\title{
Curvas preliminares de índice de sitio para bosques puros y mixtos de Nothofagus alpina y Nothofagus obliqua en la Patagonia Argentina
}

\author{
Preliminary site index curves for pure and mixed forests \\ of Nothofagus alpina and Nothofagus obliqua in Patagonia Argentina
}

\author{
Hernán Attis Beltrán a,d*, Luis Mario Chauchard a,b, Guillermo Martínez Pastur, c,d \\ * Autor de correspondencia: ${ }^{a}$ Universidad Nacional del Comahue, Sede San Martín de los Andes (AUSMA), \\ Pasaje de la Paz 235 (8370) San Martin de los Andes, Neuquén, Argentina, hattisbeltran@gmail.com \\ ${ }^{\mathrm{b}}$ Administración de Parques Nacionales (APN), Argentina. \\ ${ }^{\mathrm{c}}$ Centro Austral de Investigaciones Científicas (CADIC), Argentina. \\ ${ }^{\text {d }}$ Consejo Nacional de Investigaciones Científicas y Técnicas (CONICET), Argentina.
}

\begin{abstract}
SUMMARY
The growth of the dominant height of the stand and the IS is assumed to express the effects of all relevant site factors on the growth. The objective was to develop a function which could express the dominant growth pattern in height within the range of ages and site qualities in mixed N. alpina and N. obliqua (Neuquén, Argentina). Data were obtained from stem analyses by destructive sampling, and Chapman-Richards function was used (guide curve method). A global setting for each species (specific models) and another with both species together (mixed model) were performed. Adjustments were evaluated through standard error of the estimate, the average absolute error, the coefficient of determination; besides considering the biological behavior using a graphical tree-to-tree evaluation of the goodness of fit. A graphical analysis of the dispersion of the parameters was performed by site class for each species according to the IS. Linear models were tested to detect trends in the same. Finally, we have established four classes of site quality. The growth pattern of $N$. obliqua and N. alpina has been significantly anamorphic among the age ranges and site quality ranges studied. Species have shown similar patterns, reinforcing the similarity found in previous studies in other dimensional variables. This has facilitated the development of a unique model, which facilitates assessment of the quality of site planning and management of mixed stands.
\end{abstract}

Key words: Chapman-Richards, stem analyses, anamorphic models, Nothofagus nervosa.

\section{RESUMEN}

El crecimiento de la altura dominante y el índice de sitio (IS) de un rodal, asume que expresa los efectos de todos los factores relevantes del sitio. El objetivo fue desarrollar un modelo de altura dominante que exprese el patrón de crecimiento dentro del rango de calidades de sitio y edades en bosques mixtos de Nothofagus alpina y N. obliqua (Neuquén, Argentina). Los datos fueron obtenidos a partir de análisis fustales a través de muestreos destructivos, con los que se ajustó la función de Chapman-Richards, (método de la curva guía). Se realizó un ajuste global para cada especie(modelos específicos) y otro con ambas especies juntas (modelo mixto). Los ajustes se evaluaron a través del el error estándar de la estimación, el error absoluto medio, el coeficiente de determinación, además de considerar el comportamiento biológico mediante una evaluación gráfica árbol a árbol de la bondad del ajuste. Se realizó un análisis gráfico de la dispersión de los parámetros por clase de sitio para cada especie en función del IS, y se ensayaron modelos lineales para detectar tendencias en los mismos. Finalmente, a partir de los modelos se establecieron cuatro clases de calidad de sitio. El patrón de crecimiento de $N$. obliqua y $N$. alpina ha resultado sensiblemente anamórfico entre los rangos de edad y calidad de sitio estudiados. Las especies mostraron patrones de crecimiento similares, reforzando lo reportado en estudios anteriores para otras variables dimensionales. Esto nos permitió desarrollar un modelo único a partir de la función de crecimiento, que facilitará la evaluación de la calidad de sitio y la planificación del manejo forestal en los rodales mixtos de estas especies.

Palabras clave: Chapman-Richards, análisis fustal, modelos anamórficos, Nothofagus nervosa.

\section{INTRODUCCIÓN}

Las diferencias en las potencialidades productivas de los sitios influyen directamente en los aspectos del manejo de un rodal, entre los que se puede mencionar la definición de los objetivos productivos, el régimen silvicultural establecido, el turno de cosecha, las técnicas de aprovechamiento y la rentabilidad de la inversión (Ivancich et al. 2011). En la estrecha relación entre estos aspectos y la calidad de sitio radica la importancia y la necesidad de su evaluación. La productividad de un sitio puede evaluarse en forma indirecta a través del índice de sitio (IS), definido 
como la altura dominante alcanzada por un rodal regular a una edad determinada, llamada edad de referencia (Clutter et al. 1983, Carrero et al. 2008, Esse et al. 2014).

Para la estimación del IS suelen emplearse curvas de crecimiento en altura que expresan el patrón de crecimiento promedio de la altura de los árboles dominantes de un rodal en un sitio dado. El crecimiento de la altura dominante del rodal y por ende su IS, está relacionado con el rendimiento volumétrico total del mismo y se asume que combina y expresa los efectos de todos los factores relevantes del sitio sobre dicho crecimiento. Esta afirmación se fundamenta en la hipótesis de Eichhorn (Carrero et al. 2008, Esse et al. 2014), la cual indica que la producción total de un rodal está directamente relacionada con la altura dominante del mismo; ello permite emplearla como expresión de la potencialidad productiva del sitio. Las familias de curvas de la altura dominante expresadas a partir de una función, pueden ser proporcionales entre sí (anamórficas) o bien poseer patrones particulares para cada clase de sitio (polimórficas) (Clutter et al. 1983). Los modelos de crecimiento empleados para la planificación silvicultural y la predicción de la producción bajo diferentes esquemas silvícolas, frecuentemente emplean las funciones de crecimiento en altura dominante como funciones directrices o directoras del resto de las funciones que luego son aplicadas en el manejo forestal (Chauchard 2001, Esse et al. 2007, Esse et al. 2014), por ello son tan importantes.

En el Parque Nacional Lanín, Argentina, existe una larga tradición en el manejo forestal del bosque mixto compuesto por Nothofagus alpina Poepp. et Endl. - raulí ( $=N$. nervosa Phill (Dim. et Mil.)) y Nothofagus obliqua (Mirb.) Oerst - roble pellín, y hace aproximadamente una década se han comenzado además con ensayos de plantaciones in-situ y ex-situ. Estas especies son consideradas en la región Andino-patagónica como potenciales especies alternativas tanto para plantaciones,donde usualmente emplean especies exóticas, como para la diversificación de monocultivos de exóticas, y de esta forma promover la producción de madera de calidad aumentando el valor maderero y la conservación.

En Chile se han desarrollado índices de sitio preliminares para N. alpina, y N. obliqua (Trincado et al. 2002) y se ha modelado el desarrollo de la altura dominante de N. obliqua (Salas y García 2006). Sin embargo, en la Patagonia Argentina no se dispone de funciones que expresen los patrones de crecimiento en altura dominante de estas especies que permitan construir indicadores para la clasificación de la productividad de los sitios y el desarrollo de modelos de crecimiento para la planificación del manejo. Hay indicios de que $N$. alpina y $N$. obliqua poseen similitudes en características fisiológicas que resultan en tasas de crecimiento, longevidad, hábitos, tamaños y relaciones filogenéticas muy cercanas, y que conforman en forma pura y asociada rodales sensiblemente regulares (Ramírez y Figueroa 1987, Donoso et al. 1993, Manos 1997, Chauchard et al. 1999) y con similares ciclos de vida (Chauchard 1991, Chauchard y Sbrancia 2003). Estas similitudes entre las es- pecies insinuarían que sus crecimientos podrían ser equivalentes y con ello sería posible establecer una hipótesis de semejanza en los patrones y magnitudes de sus crecimientos.

Desarrollar herramientas con las que se pueda diagramar esquemas silvícolas teóricos, favorecerá la valorización de los beneficios productivos y ambientales del manejo tanto de los bosques naturales como de los implantados. Pero para el armado de estos esquemas silvícolas que puedan emplearse en la elaboración de los planes de manejo de los bosques naturales, como así también para establecer la potencialidad productiva de las plantaciones, es esencial poder discriminar como primera medida las calidades de los sitios (Chauchard 2001, Carrero et al. 2008). Es por ello que el objetivo de este estudio fue desarrollar una función que pueda expresar el patrón de crecimiento en altura dominante dentro del rango de calidades de sitio y edades en bosques maderables mixtos de $N$. alpina y $N$. obliqua. Se pretende responder las siguientes preguntas: (i) ¿Son similares los patrones de crecimiento de ambas especies? y (ii) ¿Es factible desarrollar un modelo alternativo común de ambas especies?

\section{MÉTODOS}

Área de estudio. El área de distribución de $N$. obliqua se localiza entre los $33^{\circ}$ y $41,5^{\circ} \mathrm{S}$, mientras que la de $N$. alpina abarca un rango latitudinal un poco más estrecho entre los $35^{\circ}$ y 40,5 $5^{\circ}$ (Donoso et al. 1993, Salas y García 2006). El muestreo se realizó dentro de las cuencas de los lagos Lácar y Nonthué (en el rango desde los $40^{\circ} 07^{\prime} \mathrm{S}$ $71^{\circ} 27^{\prime} \mathrm{O}$ hasta los $40^{\circ} 07^{\prime} \mathrm{S}-7^{\circ} 39^{\prime} \mathrm{O}$ ), cubriendo una superficie de 1.500 ha, en la cual se encuentran las unidades de ordenación forestal de la Reserva Nacional del Parque Nacional Lanín en la provincia del Neuquén, Argentina. Los bosques de la cuenca están dominados por tipos forestales puros y mixtos de Nothofagus spp. localizados entre los 600 y 1.100 m s.n.m. (Chauchard y Sbrancia 2003).

El clima en la zona de estudio es templado húmedo con temperaturas medias anuales de $9{ }^{\circ} \mathrm{C}$ y una precipitación media de $1.800 \mathrm{~mm}$ (Frugoni et al. 2005). Geológicamente, la región fue modelada por glaciares y posteriormente recubierta por cenizas volcánicas. De este proceso surgieron los suelos dominantes, desarrollados a partir de cenizas volcánicas holocénicas, pertenecientes al orden Andisoles y al tipo Udivitrandes húmico. Poseen una secuencia de horizontes O-A-Bw-C o bisecuencias de esos horizontes debido a los sucesivos depósitos de cenizas que dieron lugar a suelos enterrados (Frugoni et al. 2005).

Toma de datos. Los bosques muestreados fueron puros o mixtos de $N$. alpina y $N$. obliqua, regulares (un estrato de copa superior y uniforme, con una distribución normal y estrecha de diámetros) y de cobertura completa (con una cobertura del dosel mayor al 90\%, sin intervención humana reciente y con presencia de árboles intermedios) (Chauchard 1999). En ellos se seleccionaron individuos domi- 
nantes de $N$. alpina y $N$. obliqua sin daños en el fuste y copa, a los que se les realizaron análisis fustales por medio de muestreos destructivos. La dominancia se evaluó a través del tamaño relativo entre el árbol elegido y los circundantes teniendo en cuenta la altura total, el diámetro a la altura del pecho (DAP) y el tamaño, balance y relación de la copa respecto del resto de las dimensiones del árbol. La copa debía estar equilibrada en sus crecimientos laterales y ocupar entre el 25-40 \% de la altura total, porcentaje que dependía del tamaño del individuo y del nivel de competencia. En esta etapa se evitaron los árboles de crecimiento libre, y se constató que el ejemplar haya crecido entre competidores por la presencia del fuste limpio de ramas en la porción media inferior del tronco. Se eligieron árboles originados por semilla, evitando la elección de árboles provenientes de rebrotes de cepas, e.g. se descartaron aquellos individuos con grandes contrafuertes en la base del fuste.

A los árboles, previo al volteo, se les realizaron dos mediciones cruzadas de DAP con forcípula, la estimación de su altura total (HT) con hipsómetro láser (modelo VL5360, Haglöf, Suecia), y un croquis del mismo para tener una referencia posterior a la caída. Luego se procedió al apeo del árbol para su trozado y extracción de rodajas equivalentes a las alturas de: $0,30 \mathrm{~m} ; 1,30 \mathrm{~m} ; 3,30 \mathrm{~m}$ y a partir de esta última las siguientes se extrajeron cada tres metros hasta el ápice siguiendo el eje principal del árbol. En las rodajas se marcó la dirección del Norte para luego poder reordenarlas para las mediciones.

Previo al conteo y medición de los anillos de crecimiento se procedió a lijar las rodajas en la cara correspondiente a la altura de medición de la misma para mejorar su visualización y medición. Para la tarea se emplearon lijas de grano grueso (80) hasta llegar progresivamente a las de grano fino (360).En cada rodaja se trazaron dos diámetros en cruz, sobre los cuales se marcaron, de afuera hacia el centro, períodos de cinco años en cada radio con la ayuda de lupas de mano (x5) y de mesa (x10). Como la medición fue desde afuera hacia la médula, el primer período de crecimiento del árbol podía ser distinto a los cinco años. Posteriormente, se midieron los radios de cada diámetro para cada período establecido con precisión de medio milímetro con un calibre. Con estos cuatro valores de radios se obtuvo un valor promedio. Con los datos de edades y radios a diferentes alturas se construyeron los perfiles de los árboles para períodos de cinco años. Para el último periodo de crecimiento, en la zona del ápice, los perfiles se completaron por el método gráfico o de mano alzada, siguiendo la tendencia del perfil (Chauchard 2001).Se analizó la presencia de supresión temprana por competencia en cada árbol a través de los gráficos de dispersión de los datos altura-edad observados. Se evaluó la posibilidad de evitar su inclusión en los ajustes utilizando los datos a partir del DAP y considerando como edad cero a la establecida a 1,3m del suelo, denominándola edad al DAP (EAP). De esta manera se evita considerar en el ajuste la región de la curva que podría presentar supresión inicial del crecimiento del árbol (Thrower 1989).
Con la base de datos se realizó previamente una validación de los modelos de Salas y García (2006) y Trincado et al. (2002), estimando la altura total de los árboles en función de la edad de los mismos y teniendo en cuenta el índice de sitio. Para esta validación se emplearon el error estándar de la estimación (EEE), el error medio (EM), el error absoluto medio (EAM) y la raíz del error medio cuadrático porcentual (REMC\%).Los errores se calcularon como la diferencia de los datos observados respecto de los predichos por cada modelo.

$$
\begin{aligned}
& \mathrm{EM}=\frac{\left(y_{o}-y_{e}\right)}{n} \\
& \mathrm{EAM}=\frac{\left|y_{o}-y_{e}\right|}{n} \\
& \mathrm{REMC} \%=\frac{100}{\hat{\mathrm{y}}} \cdot \sqrt{\sum \frac{\left(y_{o}-y_{e}\right)^{2}}{n}}
\end{aligned}
$$

Modelos ajustados. La función que se empleó suele conocerse como de Chapman-Richards y es ampliamente utilizada en los estudios de crecimiento y rendimiento de masas forestales por la flexibilidad para caracterizar los patrones de los crecimientos anuales y acumulados de las especies forestales (Richards 1959, Osumi 1983, Zeide 1993, Vanclay 1994, Chauchard 2001, Salas y García 2006, Esse et al. 2007, 2014, Carrero et al. 2008, Ivancich et al. 2011).

Esta función cumple con las principales características que deben tener las curvas de crecimiento: forma sigmoidea, punto de inflexión que se puede determinar matemáticamente y crecimiento asintótico cuando la edad tiende a infinito. La función ha sido tratada en su forma tri-paramétrica, con la altura dominante como la variable de respuesta y la edad como la variable independiente o regresora [4]:

$$
\mathrm{H}_{0}=\mathrm{b}_{0}\left(1-\mathrm{e}^{-\mathrm{b}_{1} * \mathrm{~T}}\right)^{\mathrm{b}_{2}}
$$

Donde, $H_{0}$ : altura total (m) al tiempo $T$; $T$ : edad (años); y $b_{0}, b_{1}$ y $b_{2}$ : parámetros a ser ajustados de la función.

Si bien el ajuste del modelo va a depender del fenómeno a estudiar y del sistema que se desee desarrollar, es usual que uno de los parámetros de la función sea particular para cada sitio (local), mientras que los otros sean comunes a todos ellos (globales). La función [4] tiene su origen fijado en $\left(H_{o}, T_{o}\right)$ si se emplea la edad total, o $\left(H_{1,3}, T_{i}\right)$ si se emplea un desplazamiento del origen para evitar la supresión temprana.

El primer paso en el desarrollo del modelo fue el ajuste individual de la función [4] para cada uno de los árboles con el fin de evaluar la flexibilidad de la misma y obtener los parámetros individuales particulares. Posteriormente, para desarrollar el método de la curva guía, se realizó un ajuste global con todos los árboles juntos, primero para cada especie por separado y luego con ambas especies juntas (modelo mixto). Las estimaciones de los parámetros 
para los ajustes se determinaron a través de un procedimiento no lineal denominado de Marquadtcon aproximación iterativa (Draper y Smith 1981). Los parámetros para el inicio del proceso de aproximación iterativa de los coeficientes de la regresión fueron tomados de otros estudios ya realizados (Chauchard 2001, Salas y García 2006). Se evaluó gráficamente la bondad de ajuste y las capacidades predictivas de los modelos se evaluaron a través de los siguientes estadísticos: coeficiente de determinación $\left(R^{2}\right)$, el error estándar de la estimación (EEE) y el error absoluto medio (EAM). En el ajuste no lineal el $R^{2}$ es un estadístico que da una idea bastante intuitiva de la variabilidad que explica un determinado modelo y principalmente fue utilizado en los ajustes individuales. Sin embargo, le hemos dado mayor preponderancia a los estadísticos de error, a los gráficos de ajuste y a la significancia biológica.

Con el ajuste global de la curva guía se construyeron las familias o conjunto de curvas de crecimiento en altura, de modo de cubrir el rango de estudio de cada especie y de ambas en conjunto. Para ello se procedió a armonizar o indexar la función empleando el índice de sitio como una nueva variable independiente (Zepeda y Rivero 1984, Burkhart y Gregoire 1994). El periodo de evaluación de los patrones de crecimiento fue entre los 20 a 100 (edad total), que constituyen las edades de interés para el manejo forestal de estos bosques.

Para poder completar el proceso de indexación de la función se estableció la edad de referencia para definir el índice de sitio, con el cual posteriormente se establecieron las clases de sitio o de productividad de los rodales. En ciertas ocasiones se recomienda elegir edades próximas al turno (Clutter et al. 1983), aunque otros autores citan que en condiciones normales de crecimiento, en especies de crecimiento lento lo habitual es escoger la edad de referencia próxima a la mitad del turno o al culminar el incremento en altura de la especie (Zepeda y Rivero 1984, Ortega y Montero 1988).El rango de índice de sitio de estas clases de sitio se fijó tomando como base el rango de alturas que resultaron de las muestras disponibles.

Para evaluar la aptitud de la función guía indexada al expresar los patrones de crecimiento, se contrastó la misma con la curva observada de cada árbol, determinando sus precisiones y desvíos, empleando el error absoluto medio (EAM) y el error estándar de la estimación (EEE), como así también su bondad gráfica, evaluando la coherencia de la curva ajustada para expresar el curva real. Además se agruparon en cada clase las curvas de los árboles que correspondían según su índice de sitio y se realizaron confrontaciones gráficas con las curvas indexadas que se limitaban para cada clase. Se infirió el comportamiento biológico de los modelos a través de los gráficos del crecimiento en altura y del incremento medio anual (IMA) en función de la edad y la clase de calidad de sitio.

Por otro lado, para tratar de explicar el comportamiento de los parámetros, se realizó un análisis gráfico de la dispersión de los parámetros promedios por clase de sitio de cada especie en función del índice de sitio y se ensayaron modelos lineales para detectar las variaciones o tendencias de los mismos.

Evaluación del modelo mixto. Para analizar el comportamiento del modelo mixto para representar el patrón de crecimiento de cada especie, se contrastaron las salidas del mismo con las curvas observadas de los árboles de cada una de las especies estudiadas. Para evaluar las aptitudes del modelo mixto y su vinculación con cada uno de los modelos por especie se compararon estadísticamente las diferencias entre los IMA entre los 20 y los 90 años del modelo mixto, en comparación a cada una de las especies, para ello se realizó una prueba de t para muestras independientes. Las diferencias fueron graficadas en función de la edad.

\section{RESULTADOS}

Se dispusieron finalmente de 38 árboles muestras para el análisis del crecimiento en altura, de ellos 17 correspondieron a $N$. alpina y 21 a $N$. obliqua, con un rango de edades de 37 a 156 años y de alturas de 12 a 33 metros (cuadro 1). En algunas muestras se ha detectado supresión temprana (un árbol de $N$. alpina y tres árboles de $N$. obliqua), no así en la mayoría de los árboles. Con el fin de no perder las muestras, se decidió utilizar la EAP (edad a 1,3 m) y así evitar la supresión y las distorsiones en las curvas. Las edades a la cuales $N$. alpina y $N$. obliqua alcanzan la altura del pecho han sido entre 4 y 16 años, con un promedio de 10 años (figura 1). Los árboles en donde se observó supresión temprana llegaron a tardar entre 16 y 18 años en alcanzar el DAP.

El rango de edades totales de estudio se estableció entre los 30 y 100 años, de manera que al adoptar para el mismo la EAP y teniendo en cuenta el tiempo promedio registrado en alcanzar el DAP por la regeneración (figura 1), resulta en un rango de edades a la altura del pecho de entre los 20 y los 90 años. En función de ello y observando el gráfico de las curvas de crecimientos, se escogió la edad base o de referencia para la estimación del índice de sitio en 45 años de EAP. Al no observarse un patrón polimórfico de los crecimientos de las especies, se consideró que a esa edad ya se ha manifestado el potencial del sitio y se encuentra en un momento intermedio respecto de las edades de los posibles turnos, definido preliminarmente entre los 80 y 100 años (figura 2).

Validación. Confrontados los datos obtenidos con los modelos pre-existentes, se han encontrado diferencias importantes al compararlos a nivel de árbol. El modelo de Salas y García (2006) para $N$. obliqua fue el que arrojó los errores más bajos (n= 21; $\mathrm{EEE}=1,09 ; \mathrm{EM}=4,41 ; \mathrm{EAM}=4,63 \mathrm{y}$ REMC\%= 22,03) respecto de los obtenidos con el modelo de Trincado et al. (2002) para $N$. alpina $(\mathrm{n}=21 ; \mathrm{EEE}=1,68$; $\mathrm{EM}=6,37 ; \mathrm{EAM}=7,16$ y $\mathrm{REMC} \%=33)$ y $N$. obliqua $(\mathrm{n}=$ 17; $\mathrm{EEE} ; 1,36 ; \mathrm{EM}=3,88 ; \mathrm{EAM}=7,46$ y $\mathrm{REMC} \%=38,62)$. 
Cuadro 1. Datos principales del conjunto de árboles que constituyó la muestra para el estudio.

Main data of the set of trees that formed the sample for the study.

\begin{tabular}{cccccccc}
\hline Especie & $\begin{array}{c}\text { Tamaño } \\
\text { muestra }\end{array}$ & $\begin{array}{c}\text { Altura promedio (m)- } \\
\text { (desvío estándar) }\end{array}$ & $\begin{array}{c}\text { Altura } \\
\text { máxima (m) }\end{array}$ & $\begin{array}{c}\text { Altura } \\
\text { mínima (m) }\end{array}$ & $\begin{array}{c}\text { Edad al DAP promedio } \\
\text { (años)-(desvío estándar) }\end{array}$ & $\begin{array}{c}\text { Edad al } D A P \\
\text { máxima (años) }\end{array}$ & $\begin{array}{c}\text { Edad al DAP } \\
\text { mínima (años) }\end{array}$ \\
\hline N. alpina & 17 & $23,9(3,76)$ & 32,1 & 17,3 & $84,8(31,4)$ & 151 & 28 \\
N. obliqua & 21 & $21,8(6,11)$ & 33,2 & 12,7 & $75,1(22,1)$ & 111 & 41 \\
Total & 38 & $23,0(5,25)$ & 33,2 & 12,7 & $79,4(26,8)$ & 151 & 28 \\
\hline
\end{tabular}

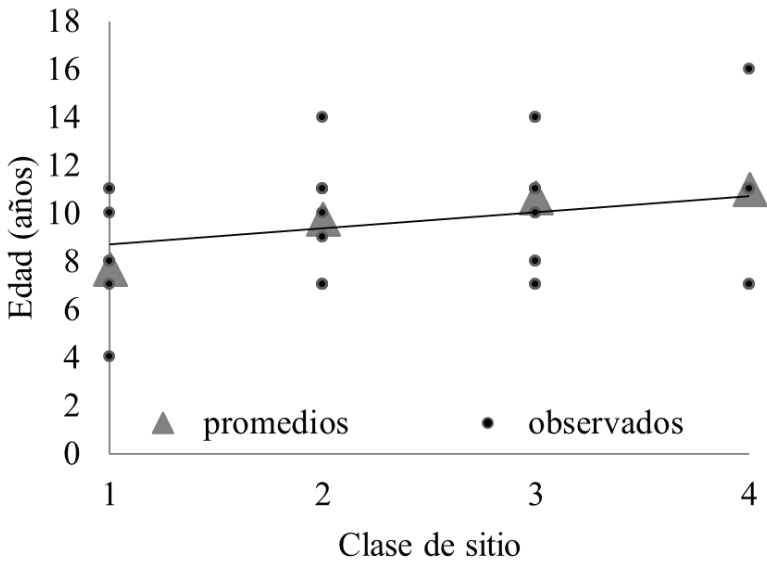

Figura 1. Años promedio en alcanzar la altura del DAP por clase de sitio.

Average years in reaching the level of DBH (diameter at breast height) for site class.

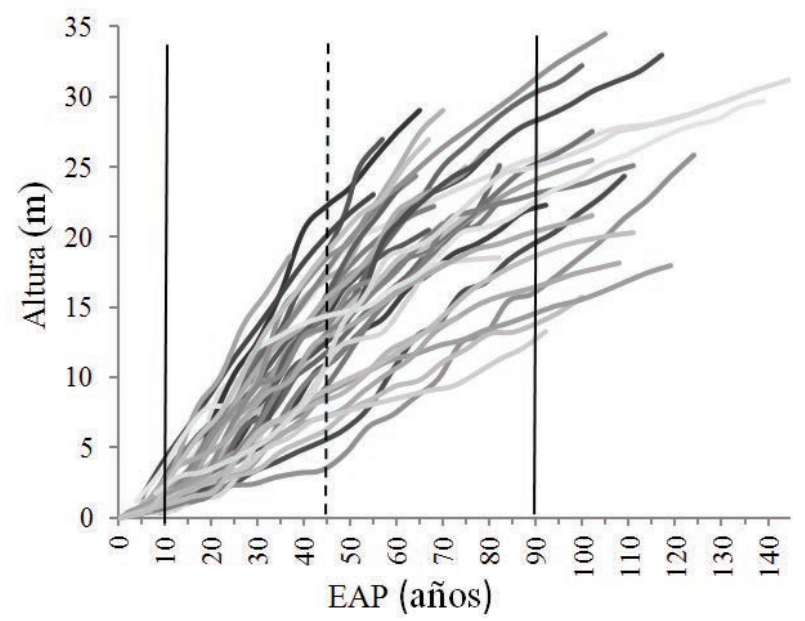

Figura 2. Curvas altura dominante-edad de los árboles de Nothofagus alpina y $N$. obliqua dentro del periodo de interés para la edad a la altura del pecho y a una EAP de referencia de 45 años.

Dominant height-age tree curves of Nothofagus alpina and $N$. obliqua within the period of interest to the age at breast height and at a reference EAP(years breast height) 45 .
Ajustes individuales. Los coeficientes de determinación $\left(R^{2}\right)$ han sido muy satisfactorios, oscilando entre $98,3 \%$ y 99,9 \%, mientras que los errores estándares de la estimación y los errores absolutos medios fueron bajos ( $\mathrm{EEE}<0,9$ y EAM $<0,7)$ y semejantes en ambas especies, inclusive en sus rangos. La amplitud en los rangos de los índice de sitio oscilaron entre $9,5 \mathrm{~m}$ y $24,0 \mathrm{~m}$ para $N$. alpina y entre $6,5 \mathrm{~m}$ y 24,0 m para $N$. obliqua.

Ajustes globales. Los estadísticos de ajuste de la curva guía o global no tienen mayor relevancia al obtenerse con los datos de todas las calidades de sitio $\left(R^{2}>65,6\right.$; $\mathrm{EEE}<4,81$; EAM $<3,78$ ). Los valores de los parámetros estimados fueron similares para los tres modelos, siendo los del modelo mixto los que tuvieron valores intermedios respecto a los de los modelos de cada especie (cuadro 2). El ajuste de la función o curva guía, con el parámetro $b_{0}$ local tuvo la mejor evaluación visual para todos los casos, al confrontar dicha curva media con todas las curvas de crecimientos individuales (figura 3).Las curvas guías mostraron comportamientos biológicos semejantes entre las especies y a su vez con el modelo mixto, que resultaron en valores similares en los crecimientos acumulados y en los incrementos medios en altura en función de la edad (figura 3). Sin embargo, se pueden observar pequeñas diferencias entre los crecimientos en altura acumulados y los respectivos incrementos medios anuales (IMA). Por ejemplo, en $N$. alpina el IMA es ligeramente superior con respecto al de $N$. obliqua a edades avanzadas (89-90 años), mientras que en el caso del modelo mixto, sus valores son intermedios a ambas especies. Los mayores IMA para ambas especias se dan aproximadamente a los 40 años (figura 3).

A partir de la curva guía, se han establecido cuatro clases de calidad de sitio, manteniendo la uniformidad entre ambas especies para facilitar la clasificación y la vinculación entre las mismas, si bien se ha encontrado que $N$. obliqua puede crecer en calidades más pobres de las que presenta $N$. alpina (figura 4). Los rangos de índice de sitio ( $\mathrm{IS}_{45}$ ) y las clase de calidad de sitio se han definido de la siguiente manera: clase I, superior, IS: >19 m; clase II, buena, IS: 19 - 15 m; clase III, regular, IS: 15 - $11 \mathrm{~m}$; clase IV, pobre, IS: $<11 \mathrm{~m}$.

Las curvas que delimitan las clases de sitio han podido contener satisfactoriamente las curvas de crecimiento de 
Cuadro 2. Parámetros y estadísticos de los ajustes globales o curvas guías para cada especie y en conjunto. $R^{2}$ : coeficiente de determinación (\%); EEE: error estándar de la estimación (m); EAM: error absoluto medio (m); $b_{0}, b_{1}, b_{2}$ : parámetros o coeficientes.

Parameters and global statistics for each species and mixed settings. $\mathrm{R}^{2}$ : coefficient of determination (\%); EEA: standard error of estimate (m); EAM: mean absolute error (m); $\mathrm{b}_{0}, \mathrm{~b}_{1}, \mathrm{~b}_{2}$ : parameters or coefficients.

\begin{tabular}{lcccccc}
\hline \multicolumn{1}{c}{ Modelo } & $b_{0}$ & $b_{1}$ & $b_{2}$ & $R^{2}$ & EEE & EAM \\
\hline [4] Nothofagus alpina & 34,3 & 0,0198 & 1,58 & 91,4 & 2,44 & 1,81 \\
[4] Nothofagus obliqua & 21,8 & 0,0256 & 1,72 & 65,6 & 4,81 & 3,78 \\
[4] Mixto & 25,6 & 0,0230 & 1,66 & 73,6 & 4,15 & 3,18 \\
\hline
\end{tabular}
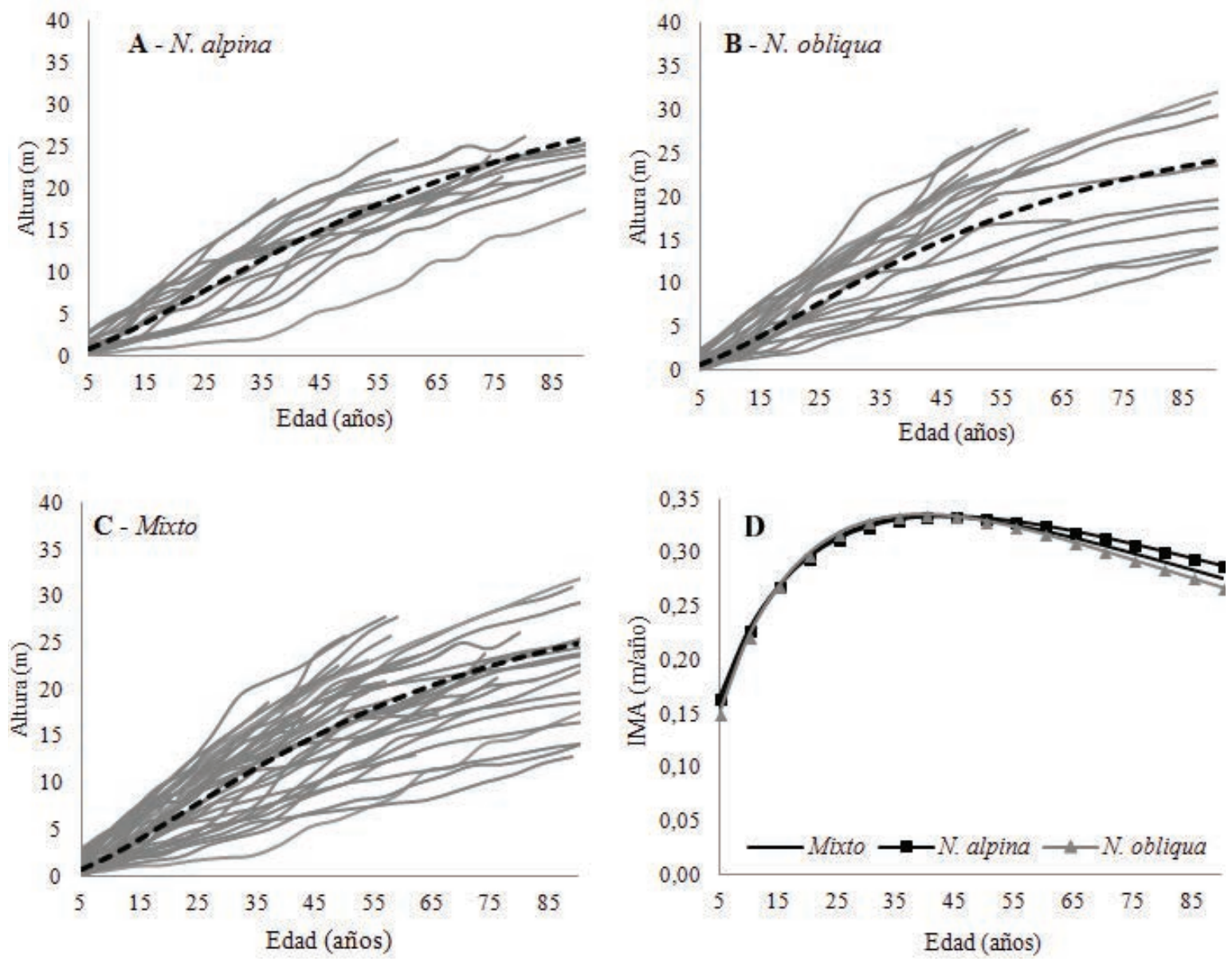

Figura 3. A, B y C: Curvas altura dominante-edad de los árboles, contrastadas con la curva guía de los modelos. D: IMA para las curva guías.

A, B and C: Dominant height-age tree curves, contrasted with the guide curve models. D: IMA (annual mean increment) for guides curve.

los árboles de la cada clase demostrando la buena aptitud de los modelos anamórficos para expresar los patrones de crecimiento (figura 4). Además, al evaluar las curvas de las clases de sitio del modelo mixto versus las curvas de los árboles, se observa también un buen comportamiento del mismo para expresar los patrones de crecimiento de cada una de las especies (figura 5).

En el análisis de los parámetros resultantes de los ajustes, se encontró una tendencia creciente del parámetro $b_{0}$ (relacionado con la asíntota de la función)con el aumento del índice de sitio para ambas especies (figura 6), siendo superior en $N$. alpina (cuadro 3 ). Con respecto a los parámetros $b_{1}$ y $b_{2}$ las tendencias de los parámetros fueron opuestas entre ambas especies. En el caso de $N$. alpina los parámetros mencionados decrecen y los de $N$. obliqua crecen con respecto al índice de sitio (figura 6).

En la comparación de las curvas de crecimiento de cada especie y por clase de sitio se observa que la diferencia de 

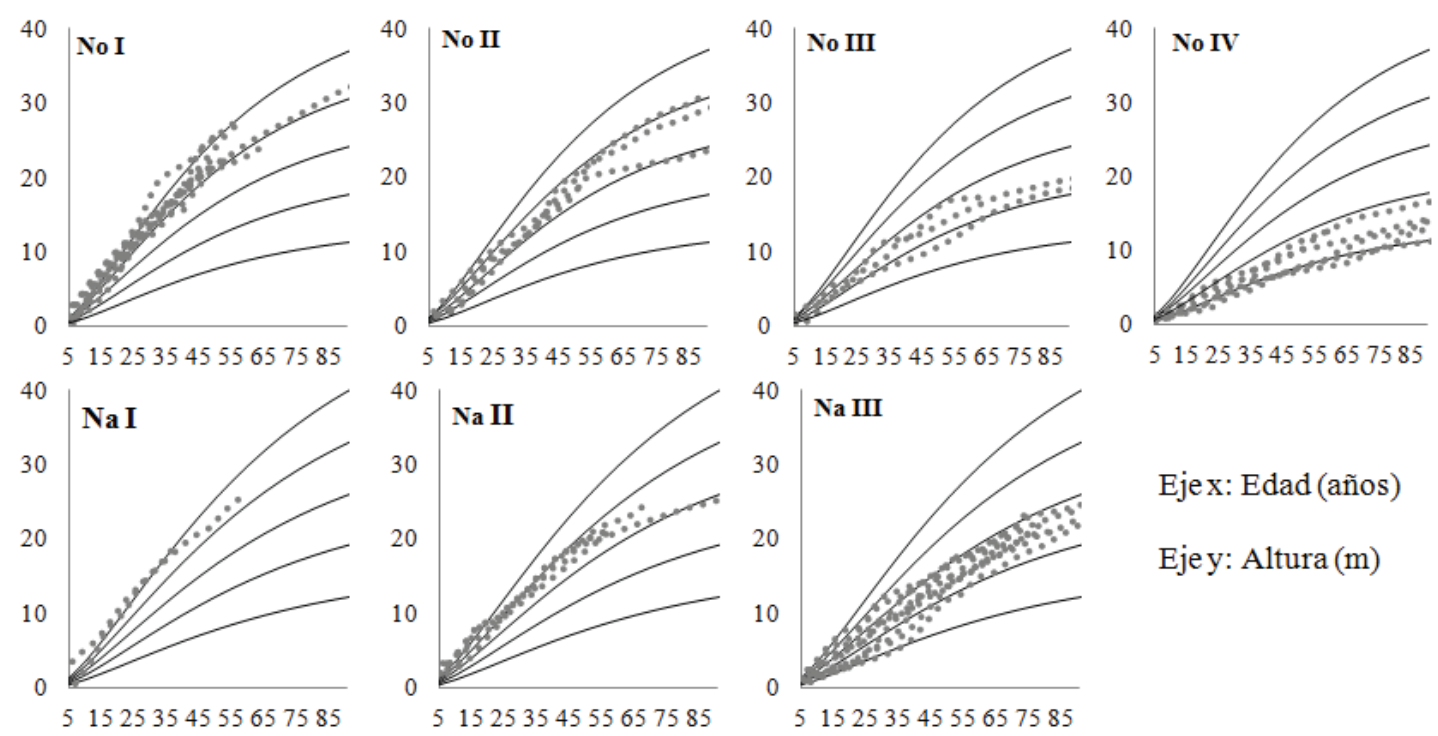

Ejex: Edad (años)

Ejey: Altura (m)

Figura 4. Familia de curvas de índice de sitio para cada especie versus los árboles de la clase. Na: Nothofagus alpina; No: N. obliqua. Los números romanos indican la clase de sitio de los árboles graficados.

Site index family curves for mixed model versus class trees. Na: Nothofagus alpina; No: N. obliqua. Roman numerals indicate the site class of the plotted trees.
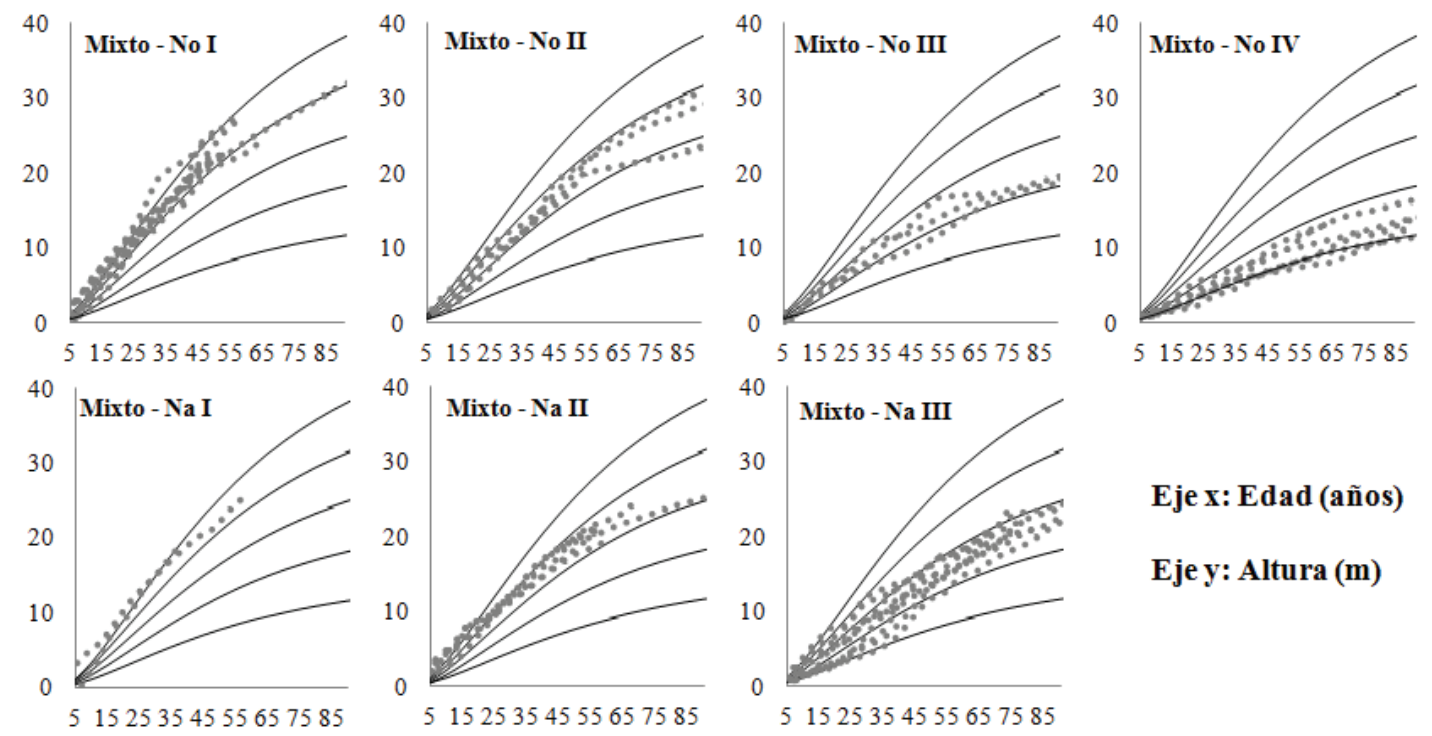

\section{Eje x: Edad (años)}

Eje y: Altura (m)

Figura 5. Familia de curvas de índice de sitio para el modelo mixto versus los árboles de la clase. Na: Nothofagus alpina; No: N. obliqua. Los números romanos indican la clase de sitio de los árboles graficados. of the plotted trees.

Site index family curves for mixed model versus class trees. Na: Nothofagus alpina; No: N. obliqua. Roman numerals indicate the site class

N. alpina por sobre $N$. obliqua a los 90 años es de $2,52 \mathrm{~m}$ en la clase de sitio superior, de 2,04 m para la clase de sitio buena, de 1,56 m para la clase de sitio regular y de 1,04 m para la clase de sitio pobre (figura 7A). Por tratarse de un modelo anamórfico las diferencias son proporcionalmente equivalentes entre las distintas clases de sitio y no superan el $8 \%$ (figura 7B). Las diferencias estadísticas entre el modelo de $N$. alpina y $N$. obliqua más altas fueron en la clase superior (Sesgo=16,79; $\mathrm{RSC}=6,8 ; \mathrm{EAM}=1,3$ ) y las más bajas en las clases inferiores ( $\left(\operatorname{sesgo}_{0}=7,20 ; \mathrm{RSC}=2,65\right.$; $\mathrm{EAM}=0,8$ ) (cuadro 3).

El comportamiento del modelo mixto con respecto a los modelos por especie fue equilibrado, no observándose diferencias entre las diferencias absolutas de las estimacio- 
Cuadro 3. Estadísticos de las diferencias entre las estimaciones del modelo de Nothofagus alpina y N. obliqua. CS: clase de sitio; RSC: suma del cuadrado de los residuos (m); EAM: error absoluto medio (m).

Statistics of the differences between the model estimates of N. alpina and N. obliqua. CS: site class; RSC: sum of squared residuals (m); EAM: mean absolute error $(\mathrm{m})$.

\begin{tabular}{cccc}
\hline CS & Sesgo & RSC & EAM \\
\hline I & 16,79 & 6,18 & 1,30 \\
II & 13,59 & 5,00 & 1,13 \\
III & 10,39 & 3,82 & 0,96 \\
IV & 7,20 & 2,65 & 0,80 \\
\hline
\end{tabular}

nes del IMA de los modelos por especie y el modelo mixto (prueba de $\mathrm{t}, \mathrm{n}=20 ; P=0,508 ; \alpha=0,05$ ). El IMA del modelo de $N$. alpina a los 90 años $0,011 \mathrm{~m}^{\text {año }}{ }^{-1}$ por encima del modelo mixto y el IMA del modelo de $N$. obliqua a los 90 años $0,008 \mathrm{~m}$ año-1 por debajo del modelo mixto (figura 8).

Los modelos finales fueron:

$$
\begin{gathered}
I S=\left(H_{0}-1,3\right) \cdot \frac{\left(1-e^{-0,019845}\right)^{1,58}}{\left(1-e^{-0,0198 . T}\right)^{1,58}} \quad \text { N. alpina } \\
I S=\left(H_{0}-1,3\right) \cdot \frac{\left(1-e^{-0,025645}\right)^{1,72}}{\left(1-e^{-0,0256 . T}\right)^{1,72}} \quad \text { N. obliqua } \\
I S=\left(H_{0}-1,3\right) \cdot \frac{\left(1-e^{-0,023045}\right)^{1,66}}{\left(1-e^{-0,0230 . T}\right)^{1,66}} \quad \text { Mixto }
\end{gathered}
$$

Donde IS: índice de sitio; $H_{0}$ : altura total (m) al tiempo $T$; $T$ : EAP (años).
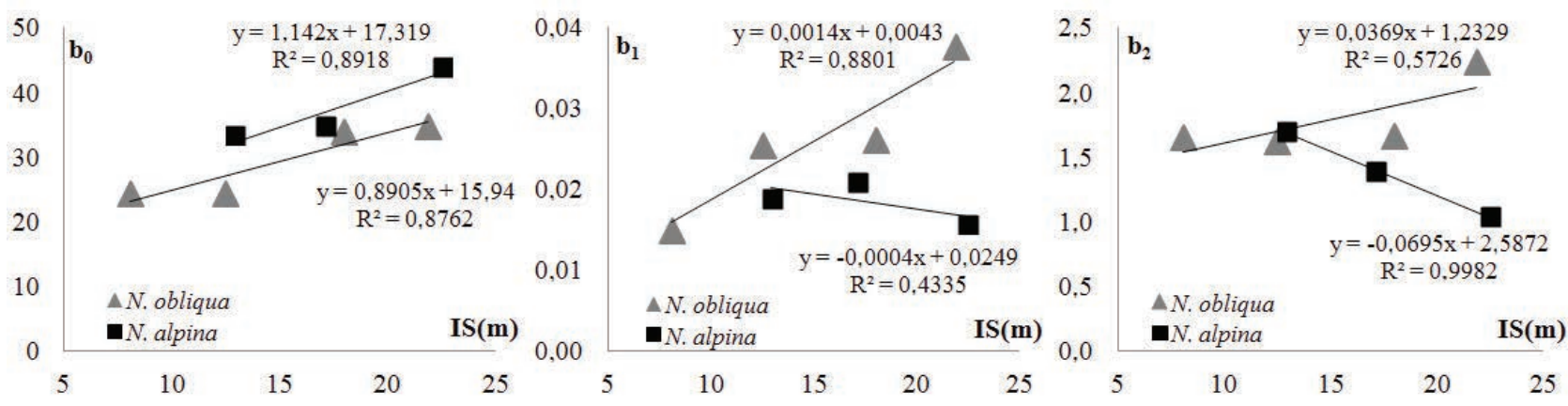

Figura 6. Distribución de los parámetros en función del índice de sitio y ajuste de modelos lineales. $b_{0}, b_{1}$ y $b_{2}$ : parámetros promedios por clase de sitio, obtenidos del ajuste individual del modelo [4]; $\mathrm{R}^{2}$ : coeficiente de determinación.

Distribution of the site index function parameters and adjust linear models. $\mathrm{b}_{0}, \mathrm{~b}_{1}$ and $\mathrm{b}_{2}$ : Average parameters by site class, obtained from the individual adjustment of model [4]; $\mathrm{R}^{2}$ : coefficient of determination.
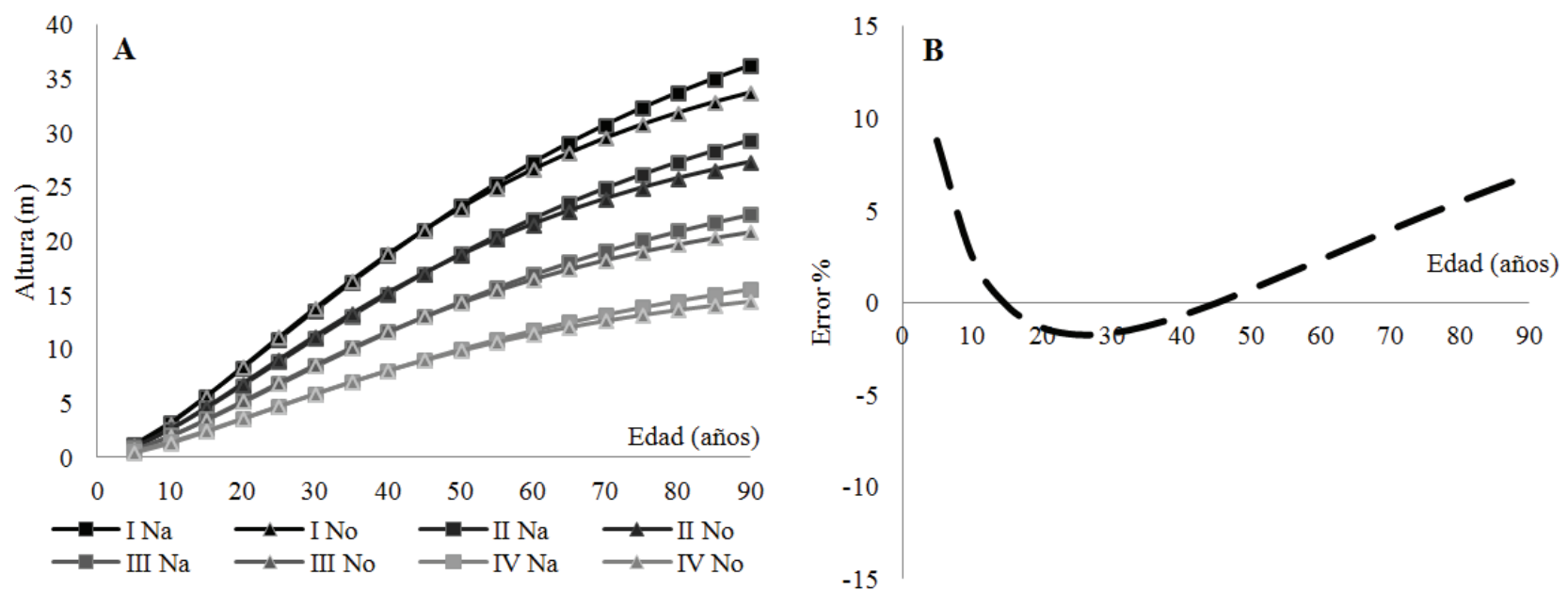

Figura 7. A) Curvas de calidad de sitio de Nothofagus alpina (Na) y N. obliqua (No). Los números romanos indican las clases de sitio. B) Diferencias porcentuales entre el modelo de N. alpina y N. obliqua.

A) Site quality Nothofagus alpina (Na) and $N$. obliqua (No) curves. Roman numerals indicate site classes. B) Percentage differences between the model of N. alpina and N. obliqua. 


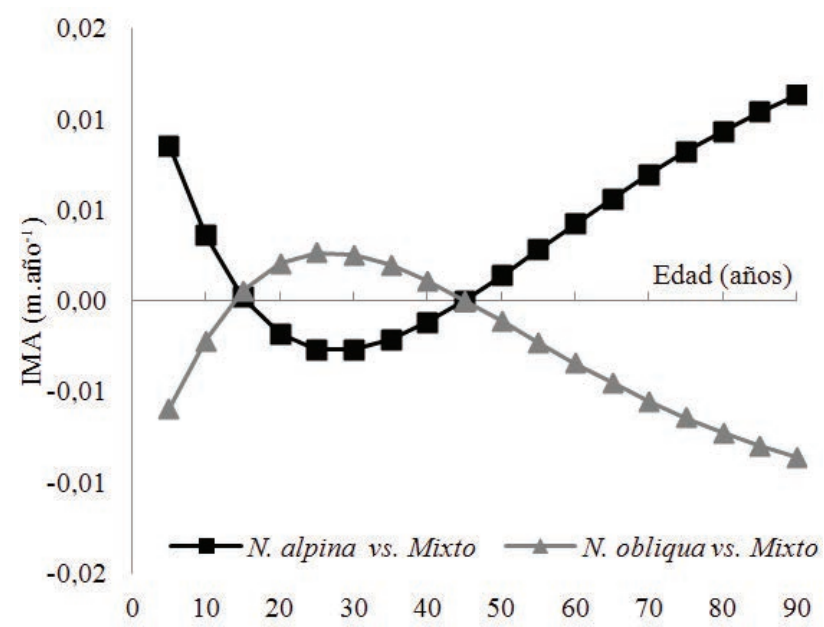

Figura 8. Diferencias en el IMA entre el modelo mixto y el de Nothofagus alpina y entre el modelo mixto y el de $N$. obliqua.

Differences among the IMA (annual mean increment) between the mixed model and Nothofagus alpina and between the mixed model and $N$. obliqua.

\section{DISCUSIÓN}

La clasificación inicial de la calidad de sitio es fundamental para diagramar esquemas silvícolas para el manejo de bosques naturales o plantaciones. Con el empleo de la altura dominante se ha podido expresar los patrones medios del crecimiento de árboles de $N$. alpina y $N$. obliqua y, con ello, establecer el índice de sitio como expresión de la potencialidad productiva. Con base biológica se han desarrollado tanto funciones de crecimiento para sendas especies, como un modelo común para las mismas, dadas las similitudes encontradas. Si bien el índice del sitio ha sido desarrollado principalmente para el estudio de una sola especie (Clutter et al. 1983, Ivancich et al. 2011), la homogeneidad de los sitios, producto de la existencia de similitudes entre las variables dimensionales y patrones de los crecimientos entre las especies estudiadas con iguales periodos de vida (Chauchard 1991, Donoso et al. 1993, Manos 1997, Chauchard et al. 1999, Chauchard y Sbrancia 2003) ha permitido la utilización de esta metodología en estos tipos de bosques mixtos que forman una cohorte.

El tamaño de las muestras de ambas especies es limitado, pero se puede considerar adecuado para realizar los estudios preliminares de evaluación de las calidades de sitio y representar los patrones de crecimiento de las especies en las áreas de estudio. Además, el estudio cobra valor por encontrarse casi la totalidad del bosque mixto dentro de Áreas Protegidas, que dificulta la obtención de muestras (Parque Nacional Lanín). El caso de la amplitud de sitios muestreados se considera más limitado para $N$. alpina (figura 3 ). Sin embargo, al encontrar similitudes en los patrones de crecimientos, tal debilidad se vería compensada al considerar ambas muestras en conjunto, por la mejor amplitud de los sitios muestreados de N. obliqua.
En algunos árboles se encontraron severas supresiones tempranas del crecimiento por competencia, que se han logrado evitar realizando los ajustes con la edad a la altura del pecho. Existe un antecedente del empleo de la EAP para estudios de crecimientos en altura de $N$. obliqua, realizado por Salas y García (2006) en Chile y para N. antarctica (Ivancich et al. 2011) y N. pumilio (Martínez Pastur et al. 1997) en Argentina. Con ello se logró disponer de la totalidad de árboles de la muestra. Si bien tal situación ha impedido emplear la edad real como variable de referencia, que es importante para algunos fines de la gestión forestal, esto se puede solucionar mediante la adición de una estimación independiente de años para alcanzar la altura del pecho, y adecuada a cada situación particular. Sin considerar los árboles con supresión temprana, hemos registrado para $N$. obliqua una edad media de 11 años para alcanzar 1,3 metros de altura, mientras que para $N$. alpina observamos una edad media de ocho años, sin encontrar evidencia de la influencia de la calidad de sitio en ello. Por lo que es posible generalizar una edad promedio de 10 años para ambas especies y todas las clases de sitio. Esto estaría indicando la poca influencia de las calidades de sitios en los crecimientos iniciales de estas especies, sin considerar la historia de vida particular de cada individuo. En un bosque secundario en Chile las edades registradas para alcanzar 1,3 metros fueron similares, con una media para $N$. obliqua fue de 9,2 años, pero con un mínimo de dos años y un máximo de 18 años (Salas y García 2006). Cabe señalar que, incluso con la utilización de la edad a la altura del pecho, pueden introducirse sesgos en las predicciones de altura-edad debido al hecho de que los árboles que son dominantes en el momento de muestreo pueden no haber sido dominantes en los años iniciales. Esto podría aumentar la pendiente de las curvas estimadas, aunque la magnitud del sesgo es incierta (Salas y García 2006).

La edad base o de referencia para este estudio se ha adoptado a 45 años a la altura del pecho, que representaría unos 55 años de edad real. La fijación de esta edad próxima a la mitad del turno tiene como ventaja el poder estimar con mejor precisión el IS en masas jóvenes (Ortega y Montero 1988, Chauchard 2001) y además a esas edades los árboles ya expresaron el potencial de crecimiento en altura del sitio.

Los modelos de Salas y García (2006) y Trincado et al. (2002) han presentado diferencias mayores en la estimación de la altura de los árboles para el área de estudio en comparación a los modelos ajustados. Probablemente las edades a estimar en el presente estudio excedían los rangos de aplicación de los modelos de Trincado et al. (2002), y la edad de referencia de 20 años adoptada por estos autores es demasiado baja teniendo en cuenta la supresión temprana observada. Con respecto al modelo de Salas y García (2006) los errores podrían deberse a patrones de crecimiento diferenciales, podrían ser adjudicados a las características propias de los sitios de estudio. Otra posibilidad que explique las diferencias podría ser la utilización de un modelo con una asíntota común, lo que, desde la óptica de la metodología empleada aquí, se aleja de lo biológicamente deseable. 
La flexibilidad de la función [4] ha permitido expresar certeramente la variación de los crecimientos en alturas de ambas especies, que demuestran ser sensiblemente anamórficos. Salas y García (2006) han empleado la misma función para expresar el crecimiento en altura dominante de $N$. obliqua, pero a diferencia del presente estudio que se ha definido a $b_{o}$ como parámetro local, estos autores han dejado a $b_{1}$ como parámetro local y han fijado $b_{o}$ (asíntota) en función de las alturas máximas registradas en Chile en otros estudios. Para ambos casos, el parámetro vinculado con la asíntota de la función carece de una interpretación biológica importante, simplemente se intenta establecer una referencia superior para el mejor ajuste e interpretación de la función en el período de interés y estudio.

La amplitud en el rango de los índices de sitio para $N$. alpina es de 14,5 m mientras que para $N$. obliqua es de 17,5 $\mathrm{m}$, lo que permite inferir una buena variación en las calidades de sitios estudiadas. La diferencia entre los rangos de índice de sitio entre las especies se produce, principalmente, porque $N$. alpina no se establece en las calidades de sitio más pobres como ocurre con $N$. obliqua. Los bosques de $N$. obliqua cubren diferentes situaciones climáticas, con rangos más amplios de temperatura, pluviometría y topografía, pero alcanzan su mayor productividad bajo condiciones de alta pluviometría y moderadas temperaturas (Thiers et al. 2008).

Si bien los estadísticos de los ajustes globales fueron relativamente bajos, con un $\mathrm{R}^{2}$ menor al $92 \%$ para el modelo de $N$. alpina y menor al $66 \%$ para el de $N$. obliqua,en este caso estos estadísticos no tienen relevancia en cuanto a bondad pues no se busca precisión sino aptitud de la función guía y la familia de curvas que es posible construir con el patrón de crecimiento de la especie. En esta situación se ajusta una función general o global para la totalidad de árboles, de todos los rangos de edades y alturas, para todas las clases de calidad de sitio. Contrariamente a lo expresado, la alta precisión y los bajos errores de un ajuste global podrían estar indicando un defecto en la amplitud de los sitios muestreados. En este sentido entonces, se puede inferir a partir del $\mathrm{R}^{2}$ de $91,4 \%$ y los errores sensiblemente menores de $N$. obliqua respecto de $N$. alpina y el modelo mixto (cuadro 2), que su muestra tiene una baja amplitud de sitios. Tal situación podría estar subsanada al poder juntar ambas especies en un modelo mixto.

Hay autores que encontraron patrones marcadamente diferentes para las mismas especies, pero estos estudios se han centrado en crecimientos tempranos, pues han trabajado con una base de datos con una edad máxima total de 50 años (Trincado et al. 2002). En el presente trabajo se ha tratado de evitar el análisis a edades muy tempranas, pues es en ellas en las cuales suelen encontrarse patrones polimórficos (Chauchard 2001) y que podrían complicar el ajuste de las funciones para edades de interés más tardías.

Por otra parte, se han encontrado similitudes entre los crecimientos de ambas especies. Se observó que los máximos incrementos se dan a los 38 años para $N$. obliqua y a los 44 años para $N$. alpina, con muy pequeñas diferencias entre ellos, a pesar de que a partir de los 55 años comienzan a manifestarse diferencias levemente superiores, llegando a los 90 años a una diferencia de $0,02 \mathrm{~m}$ año-1. Estos valores deben considerarse solo como una referencia, pues al trabajar con modelos anamórficos, no hay culminaciones diferenciales de los IMA con las diferentes calidades de sitio. En estudios llevados a cabo en Chile (Trincado et al. 2002) se menciona que en una plantación en Chile de aproximadamente 30 años el IMA en altura de los árboles que componen el dosel superior fue de $0,50 \mathrm{~m}$ para $N$. alpina y de $0,65 \mathrm{~m}$ para $N$. obliqua. Estos resultados son similares a los obtenidos por nosotros, pero con una leve diferencia superior en $N$. obliqua de $0,10 \mathrm{~m}$.

Los patrones de crecimiento en altura son expresados por el efecto combinado de los parámetros de la función (Chauchard 2001). La similitud en el parámetro $b_{0}$ y los comportamientos opuestos en los parámetros $b_{1}$ y $b_{2}$ entre ambas especies, demuestran que los mismos están íntimamente ligados y un valor alto o bajo de determinado coeficiente puede ser compensado por el valor de otro, pudiendo resultar en salidas semejantes. El parámetro $b_{0}$, que representa la asíntota de la función, ha mostrado una tendencia más clara y relación positiva con el índice de sitio en el caso de las dos especies. De todos modos $N$. alpina presenta una relación entre $b_{0}$ y las calidades de sitio con una pendiente ligeramente mayor, lo que explica las diferencias observadas a edades avanzadas. Las tendencias opuestas de los parámetros $b_{1}$ y $b_{2}$ en función del índice de sitio entre las especies, podrían indicar distintos patrones de crecimiento entre las especies, pero al observar las curvas resultantes, la influencia de los tres parámetros no permiten observar diferencias importantes, ni en la tasa de crecimiento ni en el punto de inflexión. Esto refuerza la hipótesis de que más allá de la explicación biológica que se le pueda dar a cada parámetro, en el caso de los parámetros $b_{1}$ y $b_{2}$ prima la autocompensación de los mismos por sobre el efecto individual de cada uno en las salidas de la función.

La similitud entre los comportamientos de las especies estudiadas ha motivado que el modelo mixto haya demostrado una buena aptitud para expresar el crecimiento en altura dominante para ambas especies. El mismo se comporta como un promedio entre los modelos por especies, siendo exactamente iguales los tres modelos a los 15 y 45 años (figura 7). Luego de los 45 años surgen las mayores diferencias absolutas dadas por las diferencias existentes entre las asíntotas de cada modelo, sin llegar a ser consideradas importantes. Esta situación hace que si bien con el modelo mixto se pierde cierta precisión, ello no sea importante al momento de trabajar con el mismo en la silvicultura y manejo de estos bosques, dentro del período de edades de interés.

\section{CONCLUSIONES}

El patrón de crecimiento de $N$. obliqua y $N$. alpina resulta sensiblemente anamórfico entre las edades de 30 y 100 años y para todas las calidades de sitio estudiadas, lo que indica 
la necesidad de ajustar funciones anamórficas vinculadas funcionalmente al índice de sitio. Las especies estudiadas demuestran patrones similares, reforzando la similitud encontrada en estudios anteriores en otras variables dimensionales. Tal situación ha permitido el desarrollo de un modelo único, que facilitará la evaluación de la calidad de sitio y la planificación del manejo de rodales tanto puros como mixtos. Se recomienda, sin embargo, que a medida que se vaya aumentando el número de muestras, el sistema de índice de sitio propuesto sea revisado y eventualmente calibrado.

\section{AGRADECIMIENTOS}

Los autores agradecen la colaboración del Departamento Forestal del Parque Nacional Lanín y de la cátedra de Ordenación Forestal de la Universidad Nacional del Comahue. La investigación fue financiada por la Universidad Nacional del Comahue (Proyecto 04/S016 "Ecología y manejo del bosque mixto de Nothofagus: un avance hacia la conservación"). Finalmente, un particular agradecimiento a los evaluadores, cuyos comentarios y recomendaciones mejoraron sustancialmente la calidad del trabajo.

\section{REFERENCIAS}

Burkhart H, T Gregoire. 1994. Forest Biometrics. Handbook of Statistics, Vol. 12, Fourth Edition Mac Graw Hill. 619 p.

Carrero O, M Jerez, R Macchiavelli , G Orlandoni , J Stock . 2008. Ajuste de curvas de índice de sitio mediante modelos mixtos para plantaciones de Eucalyptus urophylla en Venezuela. Interciencia 33(4):265-272.

Chauchard L. 1991. Modelos de crecimiento individual del raulí (Nothofagus alpina (Poepp. et Endl.) Oerst). In Actas VI Jornadas Técnicas: Inventario, modelos de producción y crecimiento forestales. El dorado, Misiones, Argentina. p. 246-276.

Chauchard L. 2001. Crecimiento y producción de repoblaciones de Pinus radiata D. Don en el territorio histórico de Gipuzkoa (País Vasco). Vitoria, España. Servicio Central de Publicaciones del Gobierno Vasco. Departamento de Agricultura y Pesca $\mathrm{N}^{\mathrm{0}} 40.173$ p.

Chauchard L, R Sbrancia. 2003. Modelos de crecimiento diamétrico para Nothofagus obliqua. Bosque 24(3): 3-16.

Chauchard L, R Sbrancia, M González Peñalba, L Maresca, A Rabino. 1999. Aplicación de las leyes fundamentales de la densidad de bosques de Nothofagus: Regla de los -3/2 o ley de autorraleo. Bosque 20(2): 79-94.

Clutter JL, J Forston, L Pienaar, G Brister, R Bailey. 1983.Timber Management: A quantitative approach. New York, USA. John Wiley. 333 p.

Donoso P, C Donoso, V Sandoval. 1993. Proposición de zonas de crecimiento para renovales de roble (Nothofagus obliqua) y raulí (Nothofagus alpina) en su rango de distribución natural. Bosque 14(2): 37-55.

Draper N, H Smith. 1981. Applied regression analysis. New
York, USA. John Wiley. 673 p.

Esse CR, CO Navarro, JC Pinares. 2007. Curvas de índice de sitio para Nothofagus dombeyi en la zona preandina, provincia de Cautín, IX Región, Chile. Bosque 28(2): 142-151.

Esse C, PJ Donoso, V Gerding, C Navarro, F Encina-Montoya. 2014. Modelling dominant height and site index in different edaphoclimatic zones of Nothofagus dombeyi secondary forest in the Andes of south-central Chile. Southern Forests (3): 1-8.

Frugoni MC, A Rabino, L Chauchard. 2005. La distribución de los bosques de Nothofagus y su relación con los factores de sitio en una sub-cuenca de la Patagonia Andina. In Acta del tercer Congreso Forestal Argentino y Latinoamericano (ID 145). Corrientes. Argentina. ISSN 0329-1103. p. 89-90.

Ivancich H, G Martínez Pastur, P Peri. 2011. Modelos forzados y no forzados para el cálculo del índice de sitio en bosques de Nothofagus antarctica en Patagonia Sur. Bosque 32(2): 135-145.

Manos PS. 1997. Systematics of Nothofagus (Nothofagaceae) based on rDNA spacer sequences (ITS): taxonomic congruence with morphology and plastid sequences. American Journal of Botany 84: 1137-1155.

Martínez Pastur G, PL Peri, R Vukasovic, S Vaccaro, V Piriz Carrillo. 1997. Site index equation for Nothofagus pumilio Patagonian forest. Phyton 61(1/2): 55-60.

Ortega A, G Montero. 1988. Evaluación de la calidad de las estaciones forestales. Revisión bibliográfica. ICONA, Madrid. Ecología 2: 155-184.

Osumi S. 1983. Applicabilité de la fondion de Richards al'analyse de Croissance de l'arbre. Les colloques INRA 17:77-86.

Ramírez C, Figueroa H. 1987. Fitosociología de los e de la zona higromórfica Chilena. Bosque 8(2): 127-132.

Richards FJ. 1959. A flexible growth function for empirical use. The Journal of Experimental Botany. 10(29): 290-300.

Salas C, O García. 2006. Modelling height development of mature Nothofagus obliqua. Forest Ecology and Management 229(1-3): 1-6.

Thiers O, V Gerding, E Hildebrand. 2008. Renovales de Nothofagus obliqua en centro y Sur de Chile: Factores de sitio relevantes para su productividad. In Bava J, OA Picco, MB Pildaín, P López, I Orellana eds. Libro de actas de Eco Reuniones. Segunda Reunión sobre los Nothofagus en la Patagonia. Esquel, Chubut, Argentina. p. 255-260.

Thrower JS. 1989. Site quality evaluation using site index. Silviculture Institute of British Columbia. 11 p.

Trincado G, A Kiviste, K Von Gadow. 2002. Preliminary site index model for native roble (Nothofagus obliqua) and raulí (N. alpina) in Chile. New Zealand Journal of Forest Science 32(3): 322-333.

Vanclay JK. 1994. Modelling Forest Growth and Yield. Wallingford UK. By CAB International. 304 p.

Zeide B. 1993. Analysis of growth equations. Forest Science 39(3): 594-616.

Zepeda B, B Rivero. 1984. Construcción de curvas anamórficas de índice de sitio: Ejemplificación del método de Curva Guía. Revista Ciencia Forestal 51(9): 1-38. 
